\title{
Adiabatic Theorem in Axiomatic Quantum Field Theory
}

\author{
A. S. Schwarz
}

Moscow Institute of Physical Engineering, Moscow, USSR

Received October 8, 1973; in revised form May 15, 1974

\begin{abstract}
It is shown that Møller matrices $S_{ \pm}$and scattering matrix $S$ in axiomatic field theory can be expressed through their adiabatic analogs. In particular, it is proved under certain conditions that $S_{-}=\operatorname{sim}_{\alpha \rightarrow 0} S_{\alpha}(0,-\infty) W_{\alpha}$ where $W_{\alpha}$ is a trivial phase factor [i.e. a unitary operator of the form $\exp \frac{i}{\alpha} \int r(\boldsymbol{k}) a^{+}(\boldsymbol{k}) a(\boldsymbol{k}) d \boldsymbol{k}$ ]. Corresponding results in Hamiltonian approach are discussed.
\end{abstract}

Let $H(g)$ be a family of energy operators depending on parameter $g$, $0 \leqq g \leqq 1$, and let $h(\tau)$ be an even continuous function decreasing fast at infinity and satisfying $h(0)=1$ and $0 \leqq h(\tau) \leqq 1$ (one assumes usually $\left.h(\tau)=e^{-|\tau|}\right)$. We define an adiabatic $S$-matrix as an operator

$$
S_{\alpha}=S_{\alpha}(\infty,-\infty)=\operatorname{sim}_{t \rightarrow \infty, t_{0} \rightarrow-\infty} S_{\alpha}\left(t, t_{0}\right)
$$

where $\alpha$ is positive,

$$
S_{\alpha}\left(t, t_{0}\right)=\exp (i H(0) t) U_{\alpha}\left(t, t_{0}\right) \exp \left(-i H(0) t_{0}\right)
$$

and $U_{\alpha}\left(t, t_{0}\right)$ is a solution of the equation

$$
i \frac{\partial U_{\alpha}\left(t, t_{0}\right)}{\partial t}=H(h(\alpha t)) U_{\alpha}\left(t, t_{0}\right)
$$

with the initial condition $U_{\alpha}\left(t_{0}, t_{0}\right)=1$ (i.e. $U_{\alpha}\left(t, t_{0}\right)$ is an evolution operator for the timedependent Hamiltonian $H(h(\alpha t))]$. Adiabatic Møller matrices are defined as $S_{\alpha}(0, \pm \infty)=\operatorname{sim}_{t \rightarrow \pm \infty} S_{\alpha}(0, t)$.

The main result of the present paper is the proof of equalities connecting the scattering matrix $S$ and Møller matrices $S_{ \pm}$with their adiabatic analogs in axiomatic quantum field theory (this result can be called an adiabatic theorem). Corresponding results in a Hamiltonian approach [1] will be discussed briefly at the end of the paper. It is important to note that in constructing the adiabatic $S$-matrix we need not add counter-terms to the Hamiltonian $H$; thus the renormalization of one-particle energy and field operators does not enter in this construc- 
tion (this is an essential distinction between our result and the usual approach to the adiabatic hypothesis).

We list now the conditions of our main theorem. We shall proceed from axioms of Haag-Araki type but will not require Lorentz-invariance and microscopic locality. For simplicity we assume that there is only one type of particle and that all energy operators $H(g)$ in the family considered have the same ground state (vacuum) $\Phi$. The generalization to several particle types is simple. On the other hand the discussion of the case with vacuum polarization involves a more elaborate procedure about which some remarks will be made at the end.

Specifically we shall suppose that a $C^{*}$-algebra $\mathfrak{A}(\mathcal{O})$ of bounded operators in a Hilbert space $\mathscr{H}$ is associated with every bounded open region $\mathcal{O}$ in Euclidean space $E^{3}$. We shall consider in the space $\mathscr{H}$ the momentum operator $P$ and the energy operator $H(g)$ depending on a parameter $g$, where $0 \leqq g \leqq 1$. The operator $e^{-i \boldsymbol{P x}} A e^{i \boldsymbol{P x}}$ will be denoted by $A(\boldsymbol{x})$ and the operator $\exp (i H(g) t) A(\boldsymbol{x}) \exp (-i H(g) t)$ by $A(\boldsymbol{x}, t \mid g)$.

Our axioms are the following:

1) If $\mathcal{O}_{1} \subset \mathcal{O}_{2}$, then $\mathfrak{A}\left(\mathcal{O}_{1}\right) \subset \mathfrak{A}\left(\mathcal{O}_{2}\right)$.

2) If $A \in \mathfrak{U}(\mathcal{O})$, then $A(\boldsymbol{x}, t \mid g) \in \mathfrak{A}\left((\mathcal{O}+\boldsymbol{x})_{t}\right)$, where $\mathcal{O}+\boldsymbol{x}$ denotes the translate of $\mathcal{O}$ by the 3-vector $\boldsymbol{x}$ and $(\mathcal{O}+\boldsymbol{x})_{t}$ is $|t|$-neighbourhood of $\mathcal{O}+\boldsymbol{x}$ [i.e. $\boldsymbol{\xi} \in(\mathcal{O}+\boldsymbol{x})_{t}$ if and only if the distance $\left.\varrho(\boldsymbol{\xi}, \mathcal{O}+\boldsymbol{x}) \leqq|t|\right]$.

The operator $A(\boldsymbol{x}, t \mid g)$ is infinitely often differentiable with respect to $g$ and continuous with respect to $\boldsymbol{x}, t, g$; for every $m$

3) If

then

$$
\left\|\frac{\partial^{m}}{\partial g^{m}} A(\boldsymbol{x}, t \mid g)\right\| \leqq C_{m}\|A\| .
$$

$$
A \in \mathfrak{U}\left(\mathcal{O}_{1}\right), \quad B \in \mathfrak{A}\left(\mathcal{O}_{2}\right)
$$

$$
\|[A, B]\| \leqq C_{N} \frac{\|A\|\|B\|}{\varrho\left(\mathcal{O}_{1}, \mathcal{O}_{2}\right)^{N}}
$$

where $N$ is an arbitrary integer, $\varrho\left(\mathcal{O}_{1}, \mathcal{O}_{2}\right)$ is the distance between $\mathcal{O}_{1}$ and $\mathcal{O}_{2}$ (macroscopic locality in the sense of Araki).

4) Ground state $\Phi$ of the energy operator $H(g)$ does not depend on $g$ and satisfies $\boldsymbol{P} \Phi=H(g) \Phi=0$.

5) There exists a vector distribution $\Phi(\boldsymbol{k} \mid g)$ [one-particle state of $H(g)]$ that continuously depends on $g$ and satisfies the following conditions:

a) $H(g) \Phi(\boldsymbol{k} \mid g)=\omega(\boldsymbol{k} \mid g) \Phi(\boldsymbol{k} \mid g)$, where $\omega(\boldsymbol{k} \mid g)$ is a positive smooth function of $\boldsymbol{k}, g$ and a strictly convex function of $\boldsymbol{k}$;

b) $\boldsymbol{P} \Phi(\boldsymbol{k} \mid g)=\boldsymbol{k} \Phi(\boldsymbol{k} \mid g)$,

c) $\left\langle\Phi(\boldsymbol{k} \mid g), \Phi\left(\boldsymbol{k}^{\prime} \mid g\right)\right\rangle=\delta\left(\boldsymbol{k}-\boldsymbol{k}^{\prime}\right)$, 
d) for every $\boldsymbol{k}_{0} \in E^{3}, 0 \leqq g_{0} \leqq 1$ one can choose such an operator $A \in \mathfrak{U}(\mathcal{O})$, that

$$
\left\langle\Phi\left(\boldsymbol{k}_{0} \mid g_{0}\right), A \Phi\right\rangle \neq 0 .
$$

6) Every point $(\omega, \boldsymbol{k})$ belonging to multi-particle spectrum of operators $(H(g), \boldsymbol{P})$ [i.e. to spectrum of these operators in subspace orthogonal to $\Phi$ and $\left.\Phi(f \mid g)=\int f(\boldsymbol{k}) \Phi(\boldsymbol{k} \mid g) d \boldsymbol{k}\right]$ satisfies $\omega>\omega\left(\boldsymbol{k} \mid g_{0}\right)+\varepsilon$ if $\boldsymbol{k}, g$ are sufficiently near $\boldsymbol{k}_{0}, g_{0}$ (here $\varepsilon>0$ ).

7) If $g(\tau)$ is a smooth function, then the evolution operator $U\left(t_{1}, t_{0} \mid g(\tau)\right)$ corresponding to time-dependent Hamiltonian $H(g(\tau))$ can be constructed by means of multiplicative integral [i.e.

$$
U\left(t_{1}, t_{0} \mid g(\tau)\right)=\lim _{\Delta \tau_{i} \rightarrow 0} \prod_{i=1}^{n} \exp \left(-i H\left(g\left(\tau_{i}\right)\right)\right) \Delta \tau_{i}
$$

in the sense of norm limit].

We shall fix now a smooth even finite function $h(\tau)$ of real $\tau$ which satisfies $0 \leqq h(\tau) \leqq 1$ anywhere and $h(\tau)=1$ in some neighbourhood of the point $\tau=0$. The radius of this neighbourhood will be denoted by $\delta$ and the radius of the support of function $h(\tau)$ will be denoted by $\Delta$ [i.e. $h(\tau)=1$ for $|\tau| \leqq \delta$ and $h(\tau)=0$ for $|\tau| \geqq \Delta]$.

The evolution operator corresponding to the time-dependent Hamiltonian $H(h(\alpha \tau))$ will be denoted by $U_{\alpha}\left(t, t_{0}\right)$ [in other words $\left.U_{\alpha}\left(t, t_{0}\right)=U\left(t, t_{0} \mid h(\alpha \tau)\right)\right]$.

We shall consider the set $\mathfrak{B}$ of the operators which can be represented in the form

where

$$
A=\sum_{n=1}^{\infty} A_{n}
$$

$$
A_{n} \in \mathfrak{U}\left(\mathcal{O}_{n}\right), \quad \lim _{n \rightarrow \infty} n^{k}\left\|A_{n}\right\|=0
$$

for arbitrary $\boldsymbol{k}$ (here $\mathcal{O}_{n}$ is a sphere:

$$
\left.\mathcal{O}_{n}=\{\boldsymbol{x}|| \boldsymbol{x} \mid \leqq n\}\right) \text {. }
$$

Under the conditions listed above one can by Haag-Ruelle methods construct for each Hamiltonian $H(g)$ the Møller matrices $S_{ \pm}(g)$ as operators mapping from an "asymptotic space" $\mathscr{H}_{\text {as }}$ into $\mathscr{H}$. The asymptotic space $\mathscr{H}_{\text {as }}$ is defined as the space of the Fock representation of the CCR, its vacuum vector is denoted by $\theta$ :

$\left[b(\boldsymbol{k}), b\left(\boldsymbol{k}^{\prime}\right)\right]=\left[b^{+}(\boldsymbol{k}), b^{+}\left(\boldsymbol{k}^{\prime}\right)\right]=0, \quad\left[b(\boldsymbol{k}), b^{+}\left(\boldsymbol{k}^{\prime}\right)\right]=\delta\left(\boldsymbol{k}-\boldsymbol{k}^{\prime}\right), \quad b(\boldsymbol{k}) \theta=0$.

The scattering matrix $S(g)$ is defined by the formula $S(g)=S_{+}^{+}(g) S_{-}(g)$ and acts in $\mathscr{H}_{\text {as }}$. 
It is convenient to define the Møller matrices (for any fixed $g$ ) in the following way. Let us call an operator $B \in \mathfrak{B}$ a "regular creation operator" if it can be represented in the form

$$
B=\int \sigma(\boldsymbol{x}, t) A(\boldsymbol{x}, t) d \boldsymbol{x} d t
$$

where $\sigma \in \mathscr{S}^{1}, A \in \mathfrak{B}$ and if it satisfies the conditions $B^{+} \Phi=0, B \Phi$ is a one particle state [i.e. $B \Phi=\Phi(f)]$. Now $S_{ \pm}$are those isometric operators from $\mathscr{H}_{\text {as }}$ into $\mathscr{H}$ which for arbitrary regular creation operators $B^{(1)}, \ldots B^{(n)}$ and smooth, finite functions $\varphi_{1}(\boldsymbol{k}), \ldots, \varphi_{n}(\boldsymbol{k})$ satisfy the equality

Here

$$
\lim _{t \rightarrow \pm \infty} B^{(1)}\left(\varphi_{1}, t\right) \ldots B^{(n)}\left(\varphi_{n}, t\right)=S_{ \pm} b^{+}\left(\bar{f}_{1} \bar{\varphi}_{1}\right) \ldots b^{+}\left(\bar{f}_{n} \bar{\varphi}_{n}\right) \theta .
$$

$$
\begin{aligned}
B^{(i)}\left(\varphi_{i}, t\right) & =\int \tilde{\varphi}_{i}^{t}(\boldsymbol{x}) B^{(i)}(\boldsymbol{x}, t) d \boldsymbol{x}, \\
b^{+}\left(\bar{f}_{i} \bar{\varphi}_{i}\right) & =\int f_{i}(\boldsymbol{k}) \varphi_{i}(\boldsymbol{k}) b^{+}(\boldsymbol{k}) d \boldsymbol{k} .
\end{aligned}
$$

The functions $\tilde{\varphi}_{i}^{t}$ and $f_{i}$ are given by the formulae:

$$
\begin{aligned}
\tilde{\varphi}_{i}^{t}(\boldsymbol{x}) & =(2 \pi)^{-3} \int e^{-i \omega(\boldsymbol{k}) t+i \boldsymbol{k} \boldsymbol{x}} \varphi_{i}(\boldsymbol{k}) d \boldsymbol{k}, \\
B^{(i)} \Phi & =\Phi\left(f_{i}\right) .
\end{aligned}
$$

Following Haag-Ruelle considerations [3], [4] one can verify that operators $S_{ \pm}$exist and are defined uniquely by Eq. (2). (For details see [2].)

We can now state our main theorem.

Theorem. Under conditions 1)-7) one can prove the equalities

$$
\begin{aligned}
& S_{-}(1)=\operatorname{slim}_{\alpha \rightarrow 0} \operatorname{sim}_{t \rightarrow-\infty} U_{\alpha}(0, t) S_{-}(0) W_{\alpha}(t), \\
& S_{+}(1)=\operatorname{sim}_{\alpha \rightarrow 0} \operatorname{sim}_{t \rightarrow+\infty} U_{\alpha}(0, t) S_{+}(0) W_{\alpha}(t)
\end{aligned}
$$

where

$$
\begin{aligned}
W_{\alpha}(t) & =\exp \left(i \int r_{\alpha}(\boldsymbol{k} \mid t) b^{+}(\boldsymbol{k}) b(\boldsymbol{k}) d \boldsymbol{k}\right), \\
r_{\alpha}(\boldsymbol{k} \mid t) & =\int_{t}^{0} \omega(\boldsymbol{k} \mid h(\alpha \tau)) d \tau .
\end{aligned}
$$

By means of adiabatic Møller matrices the Eqs. (3), (4) can be represented in the form

$$
\begin{aligned}
S_{-}(1) & =\operatorname{sim}_{\alpha \rightarrow 0} S_{\alpha}(0,-\infty) S_{-}(0) \exp \left(i \int \varrho_{\alpha}(\boldsymbol{k}) b^{+}(\boldsymbol{k}) b(\boldsymbol{k}) d \boldsymbol{k}\right) \\
& =\operatorname{sim}_{\alpha \rightarrow 0} S_{\alpha}(0,-\infty) \exp \left(i \int \varrho_{\alpha}(\boldsymbol{k}) a_{\text {in }}^{+}(\boldsymbol{k}) a_{\mathrm{in}}(\boldsymbol{k}) d \boldsymbol{k}\right), \\
S_{+}(1) & =\operatorname{sim}_{\alpha \rightarrow 0} S_{\alpha}(0,+\infty) \exp \left(-i \int \varrho_{\alpha}(\boldsymbol{k}) a_{\mathrm{out}}^{+}(\boldsymbol{k}) a_{\text {out }}(\boldsymbol{k}) d \boldsymbol{k}\right),
\end{aligned}
$$

\footnotetext{
${ }^{1} \mathscr{S}$ denotes as usual the space of smooth functions decreasing faster than any power at infinity.
} 
where

$$
\begin{aligned}
\varrho_{\alpha}(\boldsymbol{k}) & =\int_{-\infty}^{0}(\omega(\boldsymbol{k} \mid h(\alpha \tau))-\omega(\boldsymbol{k} \mid 0)) d \tau=\frac{1}{\alpha} \int_{-\infty}^{0}(\omega(\boldsymbol{k} \mid h(\sigma))-\omega(\boldsymbol{k} \mid 0)) d \sigma \\
= & \lim _{t \rightarrow-\infty}\left(r_{\alpha}(k \mid t)-t \omega(\boldsymbol{k} \mid 0)\right), \\
a_{\text {in }}(\boldsymbol{k})= & S_{-}(0) b(\boldsymbol{k}) S_{-}^{-1}(0), \quad a_{\text {out }}(\boldsymbol{k})=S_{+}(0) b(\boldsymbol{k}) S_{+}^{-1}(0)
\end{aligned}
$$

- in- and out-operators corresponding to the energy operator $H(0)$.

In the proof of (3), (4) we shall use some lemmas. First, we introduce in the set $\mathfrak{B}$ a topology by means of the set of norms $\|A\|_{\lambda}$, where $\|A\|_{\lambda}$ denotes the greatest lower bound of such $C$ that the operator $A$ can be represented in the form (1) with $\left\|A_{n}\right\| \leqq C n^{-\lambda}$.

Let $T$ be a space of the piecewise continuous functions of 4 variables decreasing faster than any power, with topology defined by the set of norms

$$
\|\varphi\|_{\mu}=\sup |\varphi(\boldsymbol{x}, t)|(1+|\boldsymbol{x}|+|t|)^{\mu} .
$$

Lemma 1. If $A, B \in \mathfrak{B}, \lambda, \mu$ are complex numbers, $0 \leqq g \leqq 1,(\boldsymbol{x}, t) \in E^{4}$, $\varphi \in T, g(\tau)$ is a smooth function with values in $[0 ; 1]$, then operators $A(\boldsymbol{x}, t \mid g), \int \varphi(\boldsymbol{x}, t) A(\boldsymbol{x}, t \mid g) d \boldsymbol{x} d t$ are infinitely differentiable in $\mathfrak{B}$ with respect to $g$ and operators

$$
\begin{gathered}
\lambda A+\mu B, \quad A B, \quad A^{+}, \quad \frac{\partial^{m}}{\partial g^{m}} A(\boldsymbol{x}, t \mid g), \\
\frac{\partial^{m}}{\partial g^{m}} \int \varphi(\boldsymbol{x}, t) A(\boldsymbol{x}, t \mid g) d \boldsymbol{x} d t, \quad U\left(t_{1}, t_{0} \mid g(\tau)\right) A U\left(t_{0}, t_{1} \mid g(\tau)\right)
\end{gathered}
$$

belong to $\mathfrak{B}$ and are continuous with respect to $A, B, \varphi, g, \lambda, \mu$ in the topology of $\mathfrak{B}$ (in particular, this means that $\mathfrak{B}$ can be considered as a topological *-algebra). For arbitrary $N$ one can choose such a function $\sigma_{N}(A, B)$ continuously depending on $A, B \in \mathfrak{B}$ that the following inequality holds:

$$
\begin{aligned}
&\left\|\left[U\left(t_{1}, t_{0} \mid g(\tau)\right) A(\boldsymbol{x}) U\left(t_{0}, t_{1} \mid g(\tau)\right), B\right]\right\| \\
& \leqq \sigma_{n}(A, B) \frac{1+\left|t_{1}-t_{0}\right|^{N}}{1+|\boldsymbol{x}|^{N}},
\end{aligned}
$$

in the case $g(\tau) \equiv g$ we obtain that

$$
\|[A(\boldsymbol{x}, t \mid g), B]\| \leqq \sigma_{N}(A, B) \frac{1+|t|^{N}}{1+|\boldsymbol{x}|^{N}} .
$$

The proof of this lemma can be easily obtained from conditions 1), 2), 3), and 7).

Lemma 2. One can find such real function $\lambda(\boldsymbol{k} \mid g)$ that for every $A \in \mathfrak{B}$ the function $\left\langle e^{i \lambda(\boldsymbol{k} \mid g)} \Phi(\boldsymbol{k} \mid g), A \Phi\right\rangle$ is smooth. For arbitrary smooth 
finite function $f(\boldsymbol{k} \mid g)$ one can choose a family of regular creation operators $B$ which is smooth (infinitely often differentiable with respect to $g$ in the topology of $\mathfrak{B}$ ) and satisfies

$$
B_{g}^{f} \Phi=\int f(\boldsymbol{k} \mid g) e^{i \lambda(\boldsymbol{k} \mid g)} \Phi(\boldsymbol{k} \mid g) d \boldsymbol{k} .
$$

Let $A_{g}, A_{g}^{\prime}$ be two smooth families of operators, $A_{g}^{+} \Phi=0$. By means of inequality (6) one can prove that

where

$$
\left|\frac{\partial^{m}}{\partial g^{m}} \varrho(\boldsymbol{x} \mid g)\right| \leqq \frac{C}{1+|\boldsymbol{x}|^{N}}
$$

$$
\varrho(x \mid g)=\left\langle A_{g}^{\prime}(\boldsymbol{x}) \Phi, A_{g} \Phi\right\rangle=\left\langle\left[A_{g}^{+}, A_{g}^{\prime}(\boldsymbol{x})\right] \Phi, \Phi\right\rangle,
$$

$m$ and $N$ are arbitrary integers. One can conclude from this estimate that the function $\int e^{i \boldsymbol{k} \boldsymbol{x}} \varrho(\boldsymbol{x} \mid g) \mathrm{d} \boldsymbol{x}$ is smooth.

Let us consider the set $F$ of functions having the form $\left\langle\Phi(\boldsymbol{k} \mid g), B_{g} \Phi\right\rangle$ where $B_{g}$ is a smooth family of regular creation operators. If $r_{1}(\boldsymbol{k} \mid g) \in F$, $r_{2}(k \mid g) \in F$ then the function

$$
\overline{r_{1}(\boldsymbol{k} \mid g)} r_{2}(\boldsymbol{k} \mid g)=(2 \pi)^{-3} \int\left\langle B_{g}^{(1)}(\boldsymbol{x}) \Phi, B_{g}^{(2)} \Phi\right\rangle e^{i \boldsymbol{k} \boldsymbol{x}} d \boldsymbol{x}
$$

is smooth. Furthermore, for every $\boldsymbol{k}_{0} \in F^{3}, 0 \leqq g_{0} \leqq 1$ we can take a smooth family of regular creation operators $B_{g}^{\alpha, A}=\int \tilde{\alpha}(t, \boldsymbol{x} \mid g) A(\boldsymbol{x}, t \mid g)$ $d \boldsymbol{x} d t$, where $A$ is the operator satisfying the condition $5 \mathrm{~d}), \tilde{\alpha}(t, \boldsymbol{x} \mid g)$ is the Fourier transform of $\alpha(\omega, \boldsymbol{k} \mid g)$, the function $\alpha(\omega, \boldsymbol{k} \mid g)$ vanishes for $(\omega, \boldsymbol{k})$ belonging to the multi-particle spectrum of $H(g), \boldsymbol{P}$ and for $(\omega, \boldsymbol{k})$ in the half-space $\omega \leqq 0, \alpha\left(\omega\left(\boldsymbol{k}_{0} \mid g_{0}\right), \boldsymbol{k}_{0} \mid g_{0}\right) \neq 0$. The function $r_{0}(\boldsymbol{k} \mid g)$ corresponding to this family does not vanish for $\boldsymbol{k}=\boldsymbol{k}_{0}, g=g_{0}$. These two remarks are sufficient to prove the existence of such real function $\lambda(\boldsymbol{k} \mid g)$ that for arbitrary $r(\boldsymbol{k} \mid g) \in F$ the function $\exp (i \lambda(\boldsymbol{k} \mid g)) r(\boldsymbol{k} \mid g)$ is smooth (the proof is given in the appendix to [2]; it is based on the topology of fibre boundles). The function $\lambda(\boldsymbol{k} \mid g)$ satisfies the conditions of the lemma.

It is easily seen that from the smooth family of regular creation operators $B_{g}^{f}$ satisfying $B_{g}^{f} \Phi=\int e^{i \lambda(\boldsymbol{k} \mid g)} f(\boldsymbol{k} \mid g) \Phi(\boldsymbol{k} \mid g) d \boldsymbol{k}$ one can construct the family $B_{g}^{f \varphi}$ satisfying $B_{g}^{f \varphi} \Phi=\int e^{i \lambda(\boldsymbol{k} \mid g)} f(\boldsymbol{k} \mid g) \varphi(\boldsymbol{k} \mid g) \Phi(\boldsymbol{k} \mid g) d \boldsymbol{k}$ by means of the formula:

$$
B_{g}^{f \varphi}=\int \tilde{\varphi}(\boldsymbol{x} \mid g) B_{g}^{f}(\boldsymbol{x}) d \boldsymbol{x} .
$$

With the help of this observations one can construct the operators $B_{g}^{f}$ from the operators $B_{g}^{\alpha, A}$ if the function $f(\boldsymbol{k} \mid g)$ has sufficiently small support. Arbitrary smooth finite function $f(\boldsymbol{k} \mid g)$ can be represented as a sum of smooth functions with small supports; using this representation one can construct the operators $B_{g}^{f}$ for this function. 
Further we shall replace without change of the notation the vectorvalued distribution $\Phi(\boldsymbol{k} \mid g)$ by $\exp (i \lambda(\boldsymbol{k} \mid g)) \Phi(\boldsymbol{k} \mid g)$ [this distribution satisfies the conditions $5 \mathrm{a})-5 \mathrm{~d})$ and consequently can be considered as one-particle state].

We shall also suppose that

$$
\left\langle\Phi(\boldsymbol{k} \mid g), \frac{\partial}{\partial g} \Phi\left(\boldsymbol{k}^{\prime} \mid g\right)\right\rangle=0
$$

[one can easily see that this equality is fulfilled by the corresponding choice of the function $\lambda(\boldsymbol{k} \mid g)]$.

Lemma 3. If $h(\tau)=0$ for $\tau \leqq \alpha t_{0}\left(\right.$ i.e. $\left.t_{0} \leqq-\frac{\Delta}{\alpha}\right)$ then

$$
\begin{aligned}
U_{\alpha}\left(t, t_{0}\right) \Phi(f \mid 0)= & \exp \left(i r_{\alpha}(\boldsymbol{P} \mid t)-i r_{\alpha}\left(\boldsymbol{P} \mid t_{0}\right)\right)\left(\sum_{j=0}^{s} \alpha^{j} \xi_{j}(f \mid \alpha t)\right) \\
& +\alpha^{s+1} \eta_{s+1}\left(\alpha, f, t, t_{0}\right),
\end{aligned}
$$

where $\xi_{j}(f \mid \sigma)$ is defined by recurrence from equations

$$
\begin{aligned}
i \frac{d \xi_{j-1}(f \mid \sigma)}{d \sigma} & =(H(h(\sigma))-\omega(\boldsymbol{P} \mid h(\sigma))) \xi_{j}(f \mid \sigma), \\
\left\langle\frac{d \xi_{j}(f \mid \sigma)}{d \sigma}, \Phi\left(f^{\prime} \mid h(\sigma)\right)\right\rangle & =0, \\
\xi_{0}(f \mid \sigma) & =\Phi(f \mid h(\sigma)), \\
\xi_{j}\left(f \mid \sigma_{0}\right) & =0 \quad \text { for } \quad j \geqq 1, \sigma_{0} \leqq-\Delta,
\end{aligned}
$$

and

$$
\left\|\eta_{s+1}\left(\alpha, f, t, t_{0}\right)\right\| \leqq C,
$$

where $C$ does not depend on $t, t_{0}, \alpha$ but depends on $f$ and $s$ (here $f$ and $f^{\prime}$ are smooth finite functions).

For the proof we shall note that the vector $\Psi(t)=U_{\alpha}\left(t, t_{0}\right) \Phi(f \mid 0)$ satisfies the equation

$$
i \frac{d \Psi}{d t}=H(h(\alpha t)) \Psi(t)
$$

By the change of the variable $\sigma=\alpha t$ this equation can be represented in the form

$$
i \alpha \frac{d \Psi}{d \sigma}=H(h(\sigma)) \Psi(\sigma)
$$


We shall choose the solution of the Eq. (7) in the form

$$
\Psi(\sigma)=\exp \left(-\frac{i}{\alpha} \int_{\sigma_{0}}^{\sigma} \omega\left(P \mid h\left(\sigma^{\prime}\right)\right) d \sigma^{\prime}\right)\left[\left(\sum_{j=0}^{s} \alpha^{j} \xi_{j}(f \mid \sigma)\right)+\alpha^{s+1} \eta_{s+1}(f, \alpha \mid \sigma)\right] .
$$

The function $\eta_{s+1}(f, \alpha \mid \sigma)$ satisfies the equation

$$
i \alpha \frac{d \eta_{s+1}(f, \alpha \mid \sigma)}{d \sigma}=(H(h(\sigma))-\omega(P \mid h(\sigma))) \eta_{s+1}(f, \alpha \mid \sigma)-i \frac{d \xi_{s}(f \mid \sigma)}{d \sigma}
$$

with initial condition $\eta_{s+1}\left(f, \alpha \mid \sigma_{0}\right)=0$; from this equation and the inequality $^{2}\left\|\frac{d \xi(f \mid \sigma)}{d \sigma}\right\| \leqq$ const one can readily obtain an estimate

$$
\left\|\eta_{s+1}(f, \alpha \mid \sigma)\right\| \leqq \frac{\mathrm{const}}{\alpha}
$$

Taking into account that $\eta_{s}(f, \alpha \mid \sigma)=\xi_{s}(f \mid \sigma)+\alpha \eta_{s+1}(f, \alpha \mid \sigma)$ we see that $\left\|\eta_{s}(f, \alpha \mid \sigma)\right\| \leqq$ const.

This proves the lemma.

Lemma 4. For every smooth finite function $f$ one can find an operator $D_{\sigma, s}^{f} \in \mathfrak{B}$ which is infinitely often differentiable with respect to $\sigma$ in the topology of $\mathfrak{B}$ and satisfies $\xi_{s}(f \mid \sigma)=D_{\sigma, s}^{f} \Phi$ (here $s=0,1,2, \ldots$, $-\infty<\sigma<\infty)$.

For $s=0$ one can take $D_{\sigma, 0}^{f}=B_{h(\sigma)}^{f}$ where $B_{g}^{f}$ are operators constructed in Lemma 2. For $s>0$ we shall construct the operators $D_{\sigma, s}^{f}$ by recurrence. First consider a vector $\zeta_{s}(f \mid \sigma)$ which is defined by the equations

$$
\begin{aligned}
i \frac{d \xi_{s-1}(f \mid \sigma)}{d \sigma} & =(H(h(\sigma))-\omega(\boldsymbol{P} \mid h(\sigma))) \zeta_{s}(f \mid \sigma), \\
\left\langle\zeta_{s}(f \mid \sigma), \Phi\left(f^{\prime} \mid h(\sigma)\right)\right\rangle & =0 .
\end{aligned}
$$

This vector can be represented in the form

$$
\zeta_{s}(f \mid \sigma)=\gamma(H(h(\sigma)), \boldsymbol{P} \mid h(\sigma)) \frac{d \xi_{s-1}(f \mid \sigma)}{d \sigma}
$$

where $\gamma(\omega, \boldsymbol{k} \mid g)$ vanishes for $\omega=\omega(\boldsymbol{k} \mid g)$ and is equal to $i(\omega-\omega(\boldsymbol{k} \mid g))^{-1}$ if $\boldsymbol{k} \in \operatorname{supp} f$ and $(\omega, \boldsymbol{k})$ belongs to the spectrum of operators $(H(g), \boldsymbol{P})$ but $\omega \neq \omega(\boldsymbol{k} \mid g)$. The function $\gamma(\omega, \boldsymbol{k} \mid g)$ can be chosen smooth (infinitely often differentiable with respect to all variables) and vanishing for $|\boldsymbol{k}| \geqq$ const. By means of the Eq. (8) one can write that

$$
\zeta_{s}(f \mid \sigma)=\int \tilde{\gamma}(t, \boldsymbol{x} \mid h(\sigma)) E_{\sigma, s-1}^{f}(\boldsymbol{x}, t \mid h(\sigma)) \Phi d \boldsymbol{x} d t
$$

where $\tilde{\gamma}(t, \boldsymbol{x} \mid g)$ is the Fourier transform of $\gamma(\omega, \boldsymbol{k} \mid g), E_{\sigma, s-1}^{f}=\frac{d}{d \sigma} D_{\sigma, s-1}^{f}$.

${ }^{2}$ This inequality follows from Lemma 4. 
Applying Lemma 1 one can state that the operator

$$
F_{\sigma, s}^{f}=\int \tilde{\gamma}(t, \boldsymbol{x} \mid h(\sigma)) E_{\sigma, s-1}^{f}(\boldsymbol{x}, t \mid h(\sigma)) d \boldsymbol{x} d t
$$

belongs to the algebra $\mathfrak{B}$ and is infinitely often differentiable with respect to $\sigma$.

To complete the proof it is sufficient to note that

$$
\xi_{s}(f \mid \sigma)=\zeta_{s}(f \mid \sigma)+\Phi\left(\lambda_{\sigma, s} f \mid h(\sigma)\right)
$$

where the function $\lambda_{\sigma, s}(\boldsymbol{k})$ is defined by the equations

$$
\begin{gathered}
\int \frac{\partial \lambda_{\sigma, s}(\boldsymbol{k})}{\partial \sigma} f(\boldsymbol{k}) \overline{f^{\prime}(\boldsymbol{k})} d \boldsymbol{k}=-\left\langle\frac{d \zeta_{s}(f \mid \sigma)}{d \sigma}, \Phi\left(f^{\prime} \mid h(\sigma)\right)\right\rangle, \\
\lambda_{\sigma, s}(\boldsymbol{k})=0 \quad \text { for } \quad \sigma \leqq-\Delta .
\end{gathered}
$$

This permits us to choose the operator $D_{\sigma, s}^{f}$ as

$$
D_{\sigma, s}^{f}=F_{\sigma, s}^{f}+B_{h(\sigma)}^{\lambda \sigma, s} f .
$$

(One can prove that the function $\lambda_{\sigma, s}(\boldsymbol{k})$ is smooth and consequently the operator $D_{\sigma, s}^{f}$ is infinitely often differentiable with respect to $\sigma$.) For $\sigma \leqq-\Delta$ we obtain $D_{\varrho, s}^{f}=D_{-\Delta, s}^{f}$. For $\sigma \in[-\delta, \delta]$ one can prove by recurrence that

$$
\frac{d \xi_{s}(f \mid \delta)}{d \sigma}=0, \quad \zeta_{s}(f \mid \sigma)=0, \quad F_{\sigma, s}^{f}=0, \quad \frac{\partial \lambda_{\sigma, s}(\boldsymbol{k})}{\partial \sigma}=0, \quad D_{\sigma, s}^{f}=B_{1}^{\lambda_{\sigma, s} f} .
$$

Lemma 5. If $f(\boldsymbol{k}), \varphi(\boldsymbol{k})$ are smooth finite functions, then the vector

$$
\Gamma(t)=U_{\alpha}\left(t, t_{0}\right) S_{-}(0) W_{\alpha}\left(t_{0}\right) b^{+}(\bar{f} \bar{\varphi}) \theta,
$$

where $t_{0} \leqq-\frac{\Delta}{\alpha}$, can be represented in the form

$$
\Gamma(t)=Q_{t, s} \Phi+R_{s}(t)
$$

where

$$
\begin{aligned}
Q_{t, s} & =\int \tilde{\varphi}^{t, \alpha}(\boldsymbol{x})\left(\sum_{j=0}^{s} \alpha^{j} D_{\alpha t, j}^{f}(\boldsymbol{x})\right) d \boldsymbol{x}, \\
\tilde{\varphi}^{t, \alpha}(\boldsymbol{x}) & =(2 \pi)^{-3} \int \varphi^{t, \alpha}(\boldsymbol{k}) e^{i \boldsymbol{k} \boldsymbol{x}} d \boldsymbol{k}, \\
\varphi^{t, \alpha}(\boldsymbol{k}) & =\varphi(\boldsymbol{k}) \exp \left(i r_{\alpha}(\boldsymbol{k} \mid t)\right), \\
\left\|R_{s}(t)\right\| & \leqq C(f, \varphi) \alpha^{s+1} .
\end{aligned}
$$

Indeed, it is easily seen that

$$
\Gamma(t)=U_{\alpha}\left(t, t_{0}\right) \Phi\left(f \varphi^{t_{0}, \alpha} \mid 0\right) .
$$


Combining the assertions of Lemmas 3 and 4 we see that the vector $\Gamma(t)$ can be written in the form

$$
\Gamma(t)=\exp \left(i r_{\alpha}(\boldsymbol{P} \mid t)-i r_{\alpha}\left(\boldsymbol{P} \mid t_{0}\right)\right)\left(\sum_{j=0}^{s} \alpha^{j} D_{\alpha t, j}^{f \varphi^{t_{0}, \alpha}}\right)+R_{s}(t)
$$

To complete the proof one must use the fact that $D_{\sigma, j}^{f \varphi^{t_{0}, \alpha}}$ can be replaced by

$$
\int \tilde{\varphi}^{t_{0}, \alpha}(\boldsymbol{x}) D_{\sigma, j}^{f}(\boldsymbol{x}) d \boldsymbol{x}
$$

(for $j=0$ this fact is established in the proof of Lemma 2, for $j>0$ it follows from the analysis of the proof of Lemma 4).

From Lemma 5 and the equality $U_{\alpha}\left(t^{\prime}, t\right) U_{\alpha}\left(t, t_{0}\right)=U_{\alpha}\left(t^{\prime}, t_{0}\right)$ one can deduce that

$$
U_{\alpha}\left(t^{\prime}, t\right) Q_{t, s} \Phi=Q_{t^{\prime}, s} \Phi+R_{s}\left(t^{\prime}, t\right)
$$

where $\left\|R_{s}\left(t^{\prime}, t\right)\right\| \leqq C \alpha^{s+1}$, the constant $C$ depends on $f$ and $\varphi$.

Lemma 6. Let $\varphi(\boldsymbol{k})$ be a smooth finite function. Then

$$
\begin{gathered}
\left|\tilde{\varphi}^{t, \alpha}(\boldsymbol{x})\right| \leqq C|t|^{-3 / 2}, \\
\int\left|\tilde{\varphi}^{t, \alpha}(\boldsymbol{x})\right| d \boldsymbol{x} \leqq C\left(1+|t|^{3 / 2}\right) .
\end{gathered}
$$

If $\varrho\left(x, V_{\alpha}^{t} \varphi\right) \geqq \varepsilon|t|$, then

$$
\left|\tilde{\varphi}^{t, \alpha}(\boldsymbol{x})\right| \leqq \frac{C_{N, \varepsilon}}{1+|t|^{N}}
$$

Here $\tilde{\varphi}^{t, \alpha}(\boldsymbol{x})$ is defined by formula (9), $N$ is an arbitrary integer, $\varepsilon>0$, $C$ does not depend on $\boldsymbol{x}, t$, and $\alpha, V_{\alpha}^{t}(\varphi)$ denotes the set of points which can be represented in the form $-\operatorname{grad} r_{\alpha}(\boldsymbol{k} \mid t)$, where $\boldsymbol{k} \in \operatorname{supp} \varphi$.

The estimate (13) follows easily from the well-known equality

$$
\int e^{-i \sigma(\boldsymbol{k})} \varphi(\boldsymbol{k}) d \boldsymbol{k}=\int e^{-i \sigma(\boldsymbol{k})}\left(L^{N} \varphi\right)(\boldsymbol{k}) d \boldsymbol{k},
$$

where $L$ is an operator defined by the formula

$$
(L \chi)(\boldsymbol{k})=-\operatorname{div}(\boldsymbol{u} \chi), \quad \boldsymbol{u}=\frac{\operatorname{grad} \sigma}{|\operatorname{grad} \sigma|^{2}} .
$$

The proof of the inequality (11) is similar to that of the inequality (7) in [2]. The estimate (12) follows from the inequalities (11) and (13).

Lemma 7. Let $\varphi_{1}(\boldsymbol{k}), \varphi_{2}(\boldsymbol{k})$ be two finite functions with disjoint supports. Then there exists such $c>0$ that the distance between the sets $V_{\alpha}^{t}\left(\varphi_{1}\right)$ and $V_{\alpha}^{t}\left(\varphi_{2}\right)$ is not less than $c|t|$ (here $V_{\alpha}^{t}\left(\varphi_{i}\right)$ is the set of points having the form $-\operatorname{grad} r_{\alpha}(\boldsymbol{k} \mid t)$ where $\left.\boldsymbol{k} \in \operatorname{supp} \varphi_{i}\right)$. 
Using the strict convexity of the function $\omega(\boldsymbol{k} \mid g)$ one can say that $d^{2} \omega(\boldsymbol{k} \mid g) \geqq \lambda d \boldsymbol{k}^{2}$ where $\boldsymbol{k}$ runs over a bounded set, $\lambda$ is positive and does not depend on $\boldsymbol{k}$ and $g$ (the second differential is taken with respect to $\boldsymbol{k}$ ). Therefore

$$
d^{2} r_{\alpha}(\boldsymbol{k} \mid t) \geqq \lambda|t| d \boldsymbol{k}^{2}
$$

i.e. the second derivative of the function $r_{\alpha}(\boldsymbol{k} \mid t)$ in arbitrary direction is not less than $\lambda|t|$. In particular, we can assume that

$$
\boldsymbol{e}=\frac{\boldsymbol{k}_{1}-\boldsymbol{k}_{2}}{\left|\boldsymbol{k}_{1}-\boldsymbol{k}_{2}\right|}, \quad v(\sigma)=r_{\alpha}\left(\boldsymbol{k}_{2}+\sigma \boldsymbol{e} \mid t\right)
$$

and conclude that $v^{\prime \prime}(\sigma) \geqq \lambda|t|$. It is evident now that

$$
\begin{aligned}
\left|\operatorname{grad} r_{\alpha}\left(\boldsymbol{k}_{1} \mid t\right)-\operatorname{grad} r_{\alpha}\left(\boldsymbol{k}_{2} \mid t\right)\right| & \geqq\left|v^{\prime}\left(\left|\boldsymbol{k}_{1}-\boldsymbol{k}_{2}\right|\right)-v^{\prime}(0)\right| \\
& \geqq \lambda|t|\left|\boldsymbol{k}_{1}-\boldsymbol{k}_{2}\right| .
\end{aligned}
$$

This completes the proof.

Lemma 8. If $f_{1}(\boldsymbol{k}), f_{2}(\boldsymbol{k}), \varphi_{1}(\boldsymbol{k}), \varphi_{2}(\boldsymbol{k})$ are smooth finite functions and functions $\varphi_{1}(\boldsymbol{k})$ and $\varphi_{2}(\boldsymbol{k})$ have disjoint supports, then

where

$$
\left\|\left[U_{\alpha}(t+\tau, t) Q_{t, s}^{(1)} U_{\alpha}(t, t+\tau), \quad Q_{t+e, s}^{(2)}\right]\right\| \leqq \frac{C|\tau|^{N}}{1+|t|^{N}}
$$

$$
Q_{t, s}^{(i)}=\int \tilde{\varphi}_{i}^{t, \alpha}(\boldsymbol{x})\left(\sum_{j=0}^{s} \alpha^{j} D_{\alpha, t, j}^{f_{i}}(\boldsymbol{x})\right) d \boldsymbol{x},
$$

$C$ does not depend on $t, \tau, \varrho$, and $\alpha, N$ is an arbitrary integer, $|\varrho| \leqq|\tau|$.

The proof is based on Lemmas 6,7 and the estimate (5).

Lemma 9. Let $\varphi_{1}(\boldsymbol{k}), \ldots, \varphi_{n}(\boldsymbol{k})$ be smooth finite functions, having disjoint supports, $f_{1}(\boldsymbol{k}), \ldots, f_{n}(\boldsymbol{k})$-smooth finite functions, and $t \geqq t_{0}$, $t_{0} \leqq-\frac{\Delta}{\alpha}, t<-a \alpha^{-\varepsilon}, a>0, \varepsilon>0, s \geqq \frac{3}{2} n+1$. Then the vector

$$
\Psi_{n}(t)=U_{\alpha}\left(t, t_{0}\right) S_{-}(0) W_{\alpha}\left(t_{0}\right) b^{+}\left(\bar{f}_{1} \bar{\varphi}_{1}\right) \ldots b^{+}\left(\bar{f}_{n} \bar{\varphi}_{n}\right) \theta
$$

can be represented in the form

where

$$
\Psi_{n}(t)=Q_{t, s}^{(1)} \ldots Q_{t, s}^{(n)} \Phi+\pi(t)
$$

$$
\|\pi(t)\| \leqq C\left|t-t_{0}\right| \alpha^{2},
$$

$C$ does not depend on $t, t_{0}$ and $\alpha$.

Without loss of generality one can assume that $t$ and $t_{0}$ are integers,

$$
t_{0}=-\frac{\Delta}{\alpha} \text {. }
$$


We shall give an inductive proof of the Eq. (14). From (10) we conclude that

$$
\begin{aligned}
& \Psi_{n}(t+1)=U_{\alpha}(t+1, t) \Psi_{n}(t) \\
& \approx U_{\alpha}(t+1, t) Q_{t, s}^{(1)} \ldots Q_{t, s}^{(n-1)} U_{\alpha}(t, t+1) U_{\alpha}(t+1, t) Q_{t, s}^{(n)} \Phi \\
& \approx U_{\alpha}(t+1, t) Q_{t, s}^{(1)} \ldots Q_{t, s}^{(n-2)} U_{\alpha}(t, t+1) U_{\alpha}(t+1, t) Q_{t, s}^{(n-1)} U_{\alpha}(t, t+1) Q_{t+1, s}^{(n)} \Phi .
\end{aligned}
$$

The mistake made by the using of the Eq. (10) can be written in the form

$$
U_{\alpha}(t+1, t) Q_{t, s}^{(1)} \ldots Q_{t, s}^{(n-1)} U_{\alpha}(t, t+1) R_{s}(t+1, t)
$$

where $\left\|R_{s}(t+1, t)\right\| \leqq C \alpha^{s+1}$ and every seminorm of $Q_{t, s}^{(i)}$ in $\mathfrak{B}$ is less than $C\left(1+|t|^{3 / 2}\right)$ [the last fact follows from the inequality (12)]. Using these observations and the inequalities $|t| \leqq\left|t_{0}\right|=\frac{\Delta}{\alpha}, s \geqq \frac{3}{2} n+1$ one can prove that we made a mistake not exceeding const $\alpha^{2}$. Furthermore, with the help of Lemma 8 one can see that

$\Psi_{n}(t+1) \approx U_{\alpha}(t+1, t) Q_{t, s}^{(1)} \ldots Q_{t, s}^{(n-2)} U_{\alpha}(t, t+1) Q_{t+1, s}^{(n)} U_{\alpha}(t+1, t) Q_{t, s}^{(n-1)} \Phi$

To study the mistake one must apply besides Lemma 8 the inequality $t>-a \alpha^{-\varepsilon}$ and the estimate for $Q_{t, s}^{(i)}$ given above. We obtain that the mistake is less than const. $\alpha^{N}$, where $N$ is an arbitrary integer. Then we use the Eq. (10) and conclude that

$$
\Psi_{n}(t+1) \approx U_{\alpha}(t+1, t) Q_{t, s}^{(1)} \ldots Q_{t, s}^{(n-2)} U_{\alpha}(t, t+1) Q_{t+1, s}^{(n)} Q_{t+1, s}^{(n-1)} \Phi .
$$

Using several times the Eq. (10) and Lemma 8 we obtain that

$$
\begin{aligned}
\Psi_{n}(t+1) & \approx Q_{t+1, s}^{(n)} Q_{t+1, s}^{(n-1)} \ldots Q_{t+1, s}^{(1)} \Phi \\
& \approx Q_{t+1, s}^{(1)} Q_{t+1, s}^{(2)} \ldots Q_{t+1, s}^{(n)} \Phi .
\end{aligned}
$$

By each of transformations we make a mistake not exceeding const $\alpha^{2}$. The number of the induction's steps is equal to $\left|t-t_{0}\right|$; hence, the summary mistake is less than const. $\left|t-t_{0}\right| \alpha^{2}$. Lemma 9 is proved.

To complete the proof of our theorem we must take $t=-\frac{\delta}{\alpha}, t_{0}=-\frac{\Delta}{\alpha}$ in the statement of Lemma 9 (here $\delta$ is such a number that $h(\tau) \equiv 1$ on $[-\delta ; \delta])$. Then we can assert that

$$
\begin{aligned}
\tilde{\varphi}_{i}^{t, \alpha}(\boldsymbol{x}) & =\tilde{\varphi}_{i}^{t}(\boldsymbol{x})=(2 \pi)^{-3} \int e^{-i \omega(\boldsymbol{k} \mid 1) t+i \boldsymbol{k} \boldsymbol{x}} \varphi_{i}(\boldsymbol{k}) d \boldsymbol{k} \\
D_{-\delta, 0}^{f_{i}} & =B_{1}^{f_{i}}, \quad D_{-\delta, j}^{f_{i}}=B_{1}^{\lambda_{-}^{(i)} \delta_{j} f_{i}} \\
\Psi_{n}(t) & \approx \prod_{i=1}^{n}\left(\int \tilde{\varphi}_{i}^{t}(\boldsymbol{x})\left(\sum_{j=0}^{s} \alpha^{j} D_{-\delta, j}^{f_{i}}(\boldsymbol{x})\right) d \boldsymbol{x}\right) \Phi
\end{aligned}
$$

[the mistake in $(15)$ is less than const $\alpha$ ]. 
From the definition of the Møller matrix $S_{ \pm}$and from the Eq. (15) we see that

$$
\begin{aligned}
\lim _{\alpha \rightarrow 0} \lim _{\tau \rightarrow-\infty} U_{\alpha}(0, \tau) S_{-}(0) W_{\alpha}(\tau) b^{+}\left(\bar{f}_{1} \bar{\varphi}_{1}\right) \ldots b^{+}\left(\bar{f}_{n} \bar{\varphi}_{n}\right) \theta \\
=\lim _{\alpha \rightarrow 0} \exp \left(-i H(1) \frac{\delta}{\alpha}\right) U_{\alpha}\left(-\frac{\delta}{\alpha},-\frac{\Delta}{\alpha}\right) S_{-}(0) W_{\alpha}\left(-\frac{\Delta}{\alpha}\right) b^{+}\left(\bar{f}_{1} \bar{\varphi}_{1}\right) \ldots b^{+} \\
\cdot\left(\bar{f}_{n} \bar{\varphi}_{n}\right) \theta \\
=\lim _{\alpha \rightarrow 0} \exp \left(-i H(1) \frac{\delta}{\alpha}\right) \Psi_{n}\left(-\frac{\delta}{\alpha}\right) \\
=\lim _{t \rightarrow-\infty} \prod_{i=1}^{n}\left(\int \tilde{\varphi}_{i}^{t}(\boldsymbol{x})\left(\sum_{j=0}^{s} \alpha^{j} B_{1}^{\lambda^{(i)} \delta, j} f_{i}(\boldsymbol{x}, t \mid 1) d \boldsymbol{x}\right)\right) \Phi \\
=\lim _{t \rightarrow-\infty} \prod_{i=1}^{n}\left(\int \tilde{\varphi}_{i}^{t}(\boldsymbol{x}) B_{1}^{f_{i}}(\boldsymbol{x}, t \mid 1) d \boldsymbol{x}\right) \Phi \\
=S_{-}(1) b^{+}\left(\bar{f}_{1} \bar{\varphi}_{1}\right) \ldots b^{+}\left(\bar{f}_{n} \bar{\varphi}_{n}\right) \theta .
\end{aligned}
$$

To deduce the statement of the theorem from (16) it is sufficient to remember that when $\lim A_{n} \boldsymbol{x}=A \boldsymbol{x}$ for a dense set of vectors $\boldsymbol{x}$ and the norms of operators $A_{n}$ form a bounded set, one can assert that $A=\operatorname{sim} A_{n}$. (We proved our theorem for the operator $S_{-}$; the proof for the operator $S_{+}$is completely analogous).

Consider some generalization of the theorem proved above. We remark firstly that at the cost of the slight strengthening of the condition 7) one can weaken the requirement on the function $h(\tau)$ [it is sufficient to assume that $h(\tau)$ is an even continuous function which vanishes fast at infinity and satisfies $h(0)=1$.]. Furthermore, in the formulation of our main theorem one can consider immediately a topological algebra $\mathfrak{B}$ with properties listed in Lemma 1 in the place of family of algebras $\mathfrak{A}(\mathcal{O})$ satisfying conditions 1)-3). The theorem remains correct because the proof uses these properties and conditions 4)-7) only.

In this form the theorem can be easily extended to the case when the scattering theory is defined by the operators $H(g), \boldsymbol{P}$ and a family $\mathfrak{B}$ of unbounded operators. One of possible extensions is the following. We suppose, as previously, that a momentum operator $\boldsymbol{P}$ and a family of energy operators $H(g)$ act in Hilbert space $\mathscr{H}$. Let $D$ be a dense linear subset of $\mathscr{H}$ which is invariant with respect to operators $e^{i \boldsymbol{P x}}$ and $U\left(t_{1}, t_{0} \mid g(\tau)\right)$ and contains the common ground state $\Phi$ of operators $H(g)$. We consider a complete locally convex topological $*$-algebra $\mathfrak{B}$ which consists of operators acting in $D$ (i.e. $\mathfrak{B}$ is assumed to be a complete locally convex topological vector space of operators such that multiplication of operators and involution $A \rightarrow A^{+}$are continuous in $\mathfrak{B}$ ). 
A slight modification of our proof of Eqs. (3), (4) permits us to assert that these equalities remain correct for Møller matrices, corresponding to operators $H(g), \boldsymbol{P}$ and algebra $\mathfrak{B}$, if the conditions 1)-3) are replaced by the following ones:

a) One can find such a seminorm $p(A)$ in $\mathfrak{B}$ that $|\langle A \Phi, \Phi\rangle| \leqq p(A)$ (i.e. the function $A \rightarrow\langle A \Phi, \Phi\rangle$ is continuous);

b) for every $A \in \mathfrak{B}, 0 \leqq g \leqq 1$ and smooth function $g(\tau)$ the operators $U\left(t_{1}, t_{0} \mid g(\tau)\right) A(\boldsymbol{x}) U\left(t_{0}, t_{1} \mid g(\tau)\right)$ belong to $\mathfrak{B}$. If $F$ is a compact subset of $\mathfrak{B}, p-$ a seminorm in $\mathfrak{B}$, one can find such a seminorm $q$ in $\mathfrak{B}$ and such an integer that

$$
p\left(U\left(t_{1}, t_{0} \mid g(\tau)\right) A(\boldsymbol{x}) U\left(t_{0}, t_{1} \mid g(\tau)\right) \leqq\left(1+\left|t_{1}-t_{0}\right|^{k}+|\boldsymbol{x}|^{k}\right) q(A)\right.
$$

for $A \in F$. In particular, $A(\boldsymbol{x}, t \mid g) \in \mathfrak{B}$; we assume that $A(\boldsymbol{x}, t \mid g)$ is a continuous function of $\boldsymbol{x}, t, g$ and a smooth function of $g$ in the topology of $\mathfrak{B}$. If $A$ runs over a compact set $F \subset \mathfrak{B}$

$$
p\left(\frac{\partial^{m}}{\partial g^{m}} A(\boldsymbol{x}, t \mid g)\right) \leqq\left(1+|t|^{k}+|\boldsymbol{x}|^{k}\right) q(A)
$$

(here $p$ is arbitrary seminorm in $\mathfrak{B}, m$ is arbitrary integer, the seminorm $q$ in $\mathfrak{B}$ and the integer $k$ depend on $p, F$, and $m$ ).

$$
p([A(\boldsymbol{x}), B]) \leqq \frac{q(A) q(B)}{1+|\boldsymbol{x}|^{N}} .
$$

(Here $A, B$ run over a compact set $F \subset \mathfrak{B}, p$ is an arbitrary seminorm in $\mathfrak{B}, N$ is an arbitrary integer. The seminorm $q$ depends on $p, F$, and $N$.)

In conclusion we shall give some comments on the relationship between the scattering matrix and its adiabatic analog in the Hamiltonian approach.

Let $H$ be a translationally invariant Hamiltonian of the form $H=H_{0}+V$, where

$$
\begin{gathered}
H_{0}=\int \varepsilon(\boldsymbol{k}) a^{+}(\boldsymbol{k}) a(\boldsymbol{k}) d \boldsymbol{k}, \\
V=\sum_{m, n} \int V_{m, n}\left(\boldsymbol{k}_{1}, \ldots, \boldsymbol{k}_{m} \mid \boldsymbol{l}_{1}, \ldots, \boldsymbol{l}_{n}\right) a^{+}\left(\boldsymbol{k}_{1}\right) \ldots a^{+}\left(\boldsymbol{k}_{m}\right) \\
\cdot a\left(\boldsymbol{l}_{1}\right) \ldots a\left(\boldsymbol{l}_{n}\right) d^{m} \boldsymbol{k} d^{n} l, \\
V_{m, n}\left(\boldsymbol{k}_{1}, \ldots, \boldsymbol{k}_{m} \mid \boldsymbol{l}_{1}, \ldots, \boldsymbol{l}_{n}\right)=V_{m, n}\left(\boldsymbol{k}_{1}, \ldots, \boldsymbol{k}_{m} \mid \boldsymbol{l}_{1}, \ldots, \boldsymbol{l}_{n}\right) \\
\cdot \delta\left(\boldsymbol{k}_{1}+\cdots+\boldsymbol{k}_{m}-\boldsymbol{l}_{1}-\ldots-\boldsymbol{l}_{n}\right)
\end{gathered}
$$


$a^{+}(\boldsymbol{k}), a(\boldsymbol{k})$ satisfy CCR. We assume that $V_{m, n}$ belong to the space $\mathscr{S}$ and $\varepsilon(\boldsymbol{k})$ is a strictly convex smooth function which satisfies $\varepsilon\left(\boldsymbol{k}_{1}+\boldsymbol{k}_{2}\right)$ $<\varepsilon\left(\boldsymbol{k}_{1}\right)+\varepsilon\left(\boldsymbol{k}_{2}\right)$ and has all derivatives increasing slower than some power. The Hamiltonian $H=H_{0}+V$ is considered as a formal expression.

Firstly, we consider the case $V_{m, 0} \equiv 0$ (i.e. the case of Hamiltonian without vacuum polarization). We shall use perturbation theory, considering $V$ as a perturbation. In our case we do not obtain divergences by calculation of $e^{i H t}$; in other words one can say that the expression (17) determines in the frames of perturbation theory a self-adjoint operator in Fock space. Let us consider the operator $H_{0}+g V$ depending on parameter $g$ together with momentum operator $\boldsymbol{P}=\int \boldsymbol{k} a^{+}(\boldsymbol{k}) a(\boldsymbol{k}) d k$ and algebra $\mathfrak{B}$ of operators

$$
A=\sum_{m, n} \int f_{m, n}\left(\boldsymbol{k}_{1}, \ldots, \boldsymbol{k}_{m} \mid \boldsymbol{l}_{1}, \ldots, \boldsymbol{l}_{n}\right) a^{+}\left(\boldsymbol{k}_{1}\right) \ldots a^{+}\left(\boldsymbol{k}_{m}\right) a\left(\boldsymbol{l}_{1}\right) \ldots a\left(\boldsymbol{l}_{n}\right) d^{m} \boldsymbol{k} d^{n} \boldsymbol{l}
$$

where $f_{m, n} \in \mathscr{S}$, the sum in (18) is finite. $\mathfrak{B}$ can be provided with a topology of inductive limit: $\mathfrak{B}=\operatorname{ind} \lim \mathfrak{B}_{r}$, where $\mathfrak{B}_{r}$ is the space of operators having the form (15) with $m+n \leqq r$ in the topology induced by that of $\mathscr{S}$. The operators $H(g)=H_{0}+g V, \boldsymbol{P}$ and algebra $\mathfrak{B}$ satisfy the conditions of validity of Eqs. (3), (4) in each order of perturbation theory. Unfortunately, this observation is not sufficient to assert that Eqs. (3)-(4) hold in each order of perturbation theory. However the analysis of the proof shows that for these equalities can be proved in the frames of perturbation theory after some partial summation of perturbation series.

In the general case Hamiltonian of the form (17) defines an operator in the Fock space only after volume cut-off. In this case we can not construct objects satisfying conditions of the theorem proved above even in the frames of perturbation theory. However there exists a modification of our proof which leads to the equality

$$
S=\operatorname{sim}_{\alpha \rightarrow 0} \operatorname{sim}_{\Omega \rightarrow \infty} i_{\Omega} W_{\alpha}^{\Omega} S_{\alpha}^{\Omega} W_{\alpha}^{\Omega} i_{\Omega}^{+} .
$$

Here $S_{\alpha}^{\Omega}$ is the adiabatic $S$-matrix corresponding to the family of operators $H_{0}^{\Omega}+g V^{\Omega}$, operators $H_{0}^{\Omega}$ and $V^{\Omega}$ are obtained from $H_{0}$ and $V$ by means of periodic volume cut-off, $W_{\alpha}^{\Omega}$ is a trivial phase factor (i.e. a unitary operator of the form $\left.\exp \left(\frac{i}{\alpha}\left(C+\sum r_{\boldsymbol{k}} a_{\boldsymbol{k}}^{+} a_{\boldsymbol{k}}\right)\right)\right)$. Operators $H_{0}^{\Omega}, V^{\Omega}$, $W_{\alpha}^{\Omega}, S_{\alpha}^{\Omega}$ act in the Fock space $F_{\Omega}$ corresponding to the finite volume $\Omega$ and $i_{\Omega}$ denote the natural imbedding of the space $F_{\Omega}$ into the Fock space corresponding to infinite volume. The proof of the Eq. (19) is also based on the perturbation theory and uses partial summation of perturbation series. The first heuristic proof of the Eq. (19) was given in [1]. Methods of the present paper permit to formulate (19) as a rigorous result. 


\section{References}

1. Likhachev, V.N., Tyupkin, Yu.S., Schwarz,A.S.: Theor. Math. Phys. 2, 3 (1970); 10, 63 (1972)

2. Fateyev, V.A., Schwarz,A.S.: Theor. Math. Phys. 14, 152 (1973)

3. Haag, R.: Phys. Rev. 112, 669 (1958)

4. Ruelle, D.: Helv. Phys. Acta 35, 162 (1962)

Communicated by R. Haag

A. S. Schwarz

Moscow Institute of

Physical Engineering

Chair of Theoretical Physics

Kashirskoe Chaussee 1

Moscow M-409, USSR 